\title{
Lensing by Absorber Systems
}

\author{
Brice Ménard \\ Institute for Advanced Study, Einstein Drive, Princeton NJ08540, USA
}

\begin{abstract}
We propose a method for detecting gravitational magnification of distant sources, like quasars, due to absorber systems detected in their spectra. We first motivate the use of metal absorption lines rather than Lyman- $\alpha$ lines, then we show how to relate the observed moments of the source magnitude distribution to the mass distribution of absorbers. In order to illustrate the feasibility of the method, we use a simple model to estimate the amplitude of the effect expected for MgII absorption lines. Our model suggests that quasars behind strong $\mathrm{MgII}$ absorbers are in average brightened by -0.05 to -0.2 magnitude due to magnification. One must therefore revisit the claim that, in magnitude limited surveys, quasars with strong absorbers tend to be missed due to extinction effects. In addition to constraining the mass of absorber systems, applying our method will allow for the quantification of this bias.
\end{abstract}

\section{Introduction}

During the last decade, gravitational lensing has become an invaluable tool for constraining the mass of many different structures (exoplanets, stars, galaxies, clusters, large scale structure, etc.) and has significantly improved our knowledge of many of these systems. However, there have been very few results for absorption line systems, which is unfortunate because these objects present a number of interesting aspects: low-ionisation metal lines and strong HI lines sample the cold dense gas bound in galactic systems, independent of the galaxy luminosities. Moreover absorption-line measurements are largely insensitive to redshift and give us access to early stages of galaxy formation.

Gravitational lensing by absorbers has been investigated theoretically by a number of authors. So far all studies have focused on hydrogen line absorbers, which offer easier theoretical modeling than metal lines since complications due to gas metallicities can be avoided. For most of these investigations, lensing has been studied for its effects on quasar luminosity functions, i.e. the magnification bias, and on the observed distribution of impact parameters with respect to the center of the lenses (Pei 1995, Bartelmann \& Loeb 1996, Smette et al. 1997, Hamana et al. 2000, Le Brun et al. 2000, Maller et al. 2002, Perna et al. 2002). However, only a few authors have used this technique to study the absorber systems themselves. Several studies have used multiple quasar images to probe the extent of gas clouds (Smette et al. 1995, Kobayashi et al. 2002, Ellison et al 2004), but in the context of absorbers, gravitational lensing has rarely been used to constrain the mass properties of the lenses, i.e. the purpose for which it is usually used.

This general lack of observational results for absorber systems might originate in the preferential focus of previous studies on Lyman- $\alpha$ absorbers. Indeed, analytic models can be used more easily than for other absorption lines and strong systems like DLAs are expected to trace the inner part of dark matter halos and therefore favor gravitational magnification effects. However the quantity relevant to obtaining observational results is not simply the amplitude of lensing effects, but the signal-to-noise ratio of the detection that can be achieved. In such a case, strong Lyman- $\alpha$ absorbers might not be optimal candidates because large samples are difficult to build. 
Here we propose to focus on metal absorbers since large samples are becoming available with modern surveys like the SDSS. We present a method for extracting the signal expected from gravitational magnification and show how to use it to constrain the mass distribution of these systems. In order to demonstrate the feasibility of this technique, we use existing data on MgII systems, along with a simple lensing model to estimate the amplitude of the expected effects.

\section{Detecting the statistical magnification of quasars}

Having an observational strategy in mind, the statistical nature of the problem and the redshift ranges involved in the detection of quasar magnification motivate the use of metal lines. Contrarily to the Lyman- $\alpha$ line, they can be found at various redshifts from the ground given the large range of possible absorption wavelengths. They can therefore be selected in order to maximize the lensing efficiency. Moreover, they are known to probe a large range of galactic impact parameters, from a hundred kpc down to a few kpc. The method presented below can be applied to absorption lines like MgII, FeII, SiII, CIV, etc.

The presence of a galaxy close to the line-of-sight of a background source can modify its brightness in several ways: it can act as a gravitational lens and amplify the flux of the source, the presence of dust around the galaxy can extinct and redden the source's light and emission from the absorbing galaxy can affect the measured magnitude of the source. This can be described by

$$
\mathrm{f}(\lambda)=\mathrm{f}_{\mathrm{ref}}(\lambda) e^{-\tau\left(\frac{\lambda}{1+z_{\mathrm{a}}}\right)} \times \mu+\mathrm{f}_{\mathrm{gal}}(\lambda)
$$

where $f(\lambda)$ is the altered flux of a source behind an galaxy, $f_{\text {ref }}(\lambda)$ is the flux that would be observed without intervening system, $\tau_{\lambda}$ is the optical depth of the galaxy, $\mu$ its gravitational amplification and $f_{\text {gal }}(\lambda)$ is the flux of the absorbing galaxy that may contribute to the measured source flux. If the impact parameters are large enough or if the detector point spread function is narrow enough, the latter contribution can be neglected. In this case, we can write the magnitude change induced by the presence of an absorber as

$$
\begin{aligned}
\delta m & =m-m_{\text {ref }} \\
& =-2.5 \log (\mu)+\frac{2.5}{\log e} \tau\left(\frac{\lambda}{1+z_{\mathrm{a}}}\right) .
\end{aligned}
$$

Whereas gravitational magnification is achromatic, extinction effects strongly depend on wavelength and usually become small in near infrared bands. Below we will focus on magnification only, arguing that observations at appropriate wavelengths can isolate the lensing effects.

Let us consider an area of the sky which is large enough for the mean magnification to be close to unity. In this area, let us consider a population of sources, with a fraction of them being lensed. Let $\mathrm{N}_{\text {ref }}(m)$ be the intrinsic magnitude distribution of the the unlensed sources and let $\mathrm{N}_{\text {lens }}(m)$ be that of the lensed ones. Let $f$ be the fraction of sources behind a lens and $\mathrm{P}(\delta m)$ be the distribution of magnitude shifts induced by these systems. We then have the following relation between the magnitude number counts:

$$
\mathrm{N}_{\text {lens }}(m)=f \times \int \mathrm{N}_{\text {ref }}(m-\delta m) \mathrm{P}(\delta m) \mathrm{d}(\delta m) .
$$

Let us consider a population of quasars with absorber systems having a rest equivalent width greater than a certain threshold $W_{\text {min }}$. Let $\mathrm{N}_{\text {lens }}(m)$ be the intrinsic magnitude distribution of this quasar population, and let $\mathrm{N}_{\text {ref }}(m)$ be that of a population of random 

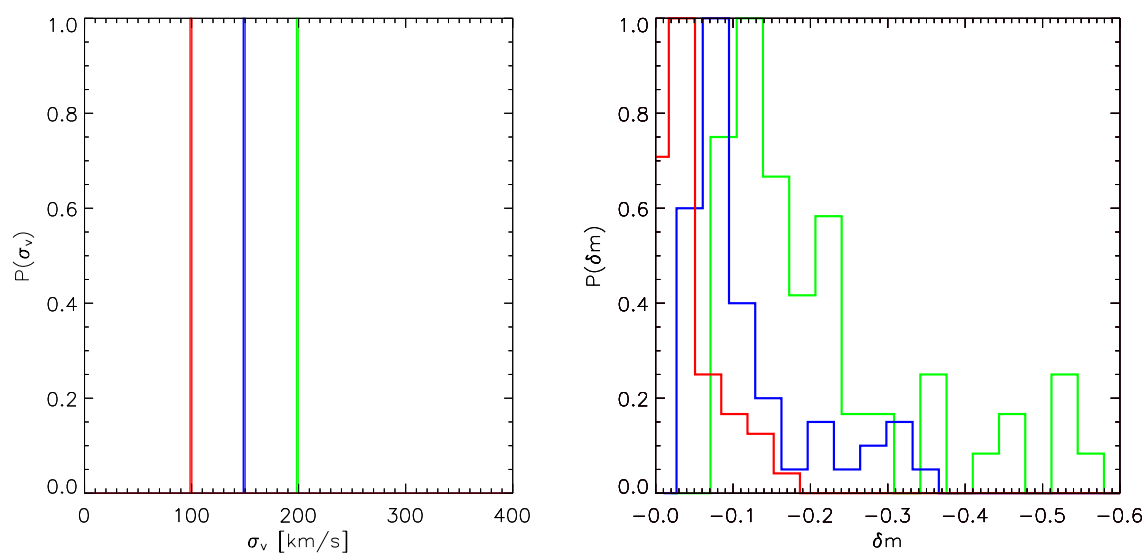

Figure 1. Left panel: velocity dispersions characterizing the MgII absorbing galaxies. Right panel: distributions of quasar magnitude shifts induced by these absorbers, considering the distribution of impact parameters measured by Steidel et al.

quasars. The quantities directly accessible to observations are

$$
\mathrm{N}_{\mathrm{ref}}^{\mathrm{obs}}(m)=\mathrm{N}_{\mathrm{ref}}(m) \mathrm{C}(m) \text { and } \mathrm{N}_{\text {lens }}^{\text {obs }}(m)=\mathrm{N}_{\text {lens }}(m) \mathrm{C}(m),
$$

where $\mathrm{C}(m)$ is the completeness of the detection procedure. In order to constrain the distribution of induced magnitude changes $\mathrm{P}(\delta m)$, which contains information on absorber masses, we propose to extract the signal by using the moments of the observed magnitude number counts. Let us first define the observed mean magnitudes of the two quasar populations as $\left\langle m_{\text {ref }}\right\rangle$ and $\left\langle m_{\text {lens }}\right\rangle$. We can now define the observable mean magnitude shift as:

$$
\Delta\left\langle m_{\text {obs }}\right\rangle=\left\langle m_{\text {lens }}\right\rangle-\left\langle m_{\text {ref }}\right\rangle
$$

As we can see by combining the previous equations, the observable quantities $\Delta\left\langle m_{\text {obs }}\right\rangle$ can be used to probe the unknown distribution $\mathrm{P}(\delta m)$. A model for this distribution can then be constrained from observations. Eq. 2.5 can be generalized to higher-order moments. It is then possible to define the likehood $\mathcal{L}(P)$ of the $n$ measured moments $\Delta\left\langle m_{\text {obs }}^{i=1, n}\right\rangle$ and minimize it in order to find the optimal parameters for $\mathrm{P}(\delta m)$, i.e. a quantity related to the distribution of absorber masses and impact parameters.

\section{A simple lensing model}

In the following, we will assume for convenience that the mass distribution of the galaxies responsible for the absorption is described by a singular isothermal sphere profile. The use of magnification effects to constrain masses requires the knowledge of the relevant halo radii. In the case of MgII systems such quantities have been measured by Steidel et al. (1994) and Steidel (1995). They used deep observations of a sample of $58 \mathrm{MgII}$ absorber systems (with a mean redshift $z=0.6$ ) in addition to a spectroscopic follow up in order to identify the galaxies responsible for the absorption. From these observations they measured the distribution of absorber rest equivalent widths $W_{0}$ as a function of galactic impact parameter $b$.

Using this measured distribution, we can first compute the characteristic magnification expected for a given absorber mass. To do so, we will consider that all absorbing galaxies are identical and we will investigate the effects of isothermal spheres with $\sigma_{v}=100,150$ 
or $200 \mathrm{~km} \mathrm{~s}^{-1}$. Considering all absorbers to be at redshift $z=0.6$, in order to match the redshifts used by Steidel et al., we can then compute the distribution of induced magnitude shifts $\delta m=-2.5 \log (\mu)$. The results are shown in Fig. 1. The left panel indicates the different velocity dispersions considered and the right panel shows the corresponding distributions of induced magnitude shifts. As can be seen, if MgII absorbers are typically surrounded by galactic dark matter halos represented by singular isothermal spheres, our calculations show that such a population will change the mean magnitude of their background quasars by -0.02 to -0.2 typically. Such changes translate into quasar flux increases of about 2 to $20 \%$. However, we recall that these induced magnitude changes are not directly observable.

The observable magnitude changes depend on the distribution of induced magnitude shifts $\mathrm{P}(\delta m)$, described in the previous section, as well as the induced quasar magnitude distribution $\mathrm{N}_{\text {ref }}(m)$ and the completeness function $\mathrm{C}(m)$ of the relevant survey. So far, the best estimate of the intrinsic quasar luminosity distribution has been been obtained by Boyle et al. (2000) and Croom et al. (2004) using the $2 \mathrm{dF}$ survey. These authors quantified the photometric and spectroscopic imcompleteness of the survey and found that the quasar magnitude distribution is well fitted by a broken power law, as measured by earlier authors. The corresponding fitting formula for the differential number counts reads (Myers et al. 2003):

$$
\mathrm{N}_{\text {ref }}(m)=\frac{N_{0}}{10^{-\alpha\left(m-m_{*}\right)}+10^{-\beta\left(m-m_{*}\right)}}
$$

with $m_{*}=19.1, \alpha=0.98$ and $\beta=0.15$. In order to take into account the completeness function, we will consider the form:

$$
\mathrm{C}(m)= \begin{cases}1 & \text { if } m<m_{b} \\ \mathrm{e}^{-\left(m-m_{b}\right)} & \text { if } m_{b}<m<m_{\text {limit }} \\ 0 & \text { otherwise }\end{cases}
$$

where $m_{\text {limit }}$ is the limiting magnitude of the survey. In order to reproduce realistic observed magnitude distributions for quasars, we will use $m_{\text {limit }}=20$ and $m_{b}=17.5$ in our numerical estimates. Note that in this case we have $\mathrm{C}(m) \approx 0$ for $m \approx m_{\text {limit }}$.

Fig. 2 shows our computation of the mean magnitude shifts for the fiducial populations of quasars and MgII absorbers, as a function of the mean absorber velocity dispersion. The left panel of the figure shows the magnitude shifts expected when ones considers the whole range of rest equivalent widths observed by Steidel et al., i.e. $0.3<W_{0}<3.3 \AA$. The dashed line shows the mean induced magnitude shift $\langle\delta m\rangle$. It ranges from -0.04 to -0.20 depending of the mean absorber velocity dispersion. The colored line shows the corresponding observable magnitude shifts $\Delta\left\langle m_{\text {obs }}\right\rangle$ for the completeness function introduced above. As can be seen, the observable effect is significantly weaker than the real mean magnitude shift induced by the population of absorbers. This is due to the fact that the quasar luminosity distribution departs only weakly from a power law. The observable shift ranges from -0.008 to -0.1 considering the range of $W_{0}$ observed by Steidel et al. The signal can be observed as a function of absorber rest equivalent width as indicated in the right panels. It can reach about -0.15 magnitude for the strongest systems.

\section{Conclusion}

We have proposed a method aimed at detecting and interpreting gravitational lensing by absorber systems. Whereas earlier studies focused on strong Lyman- $\alpha$ systems, we 


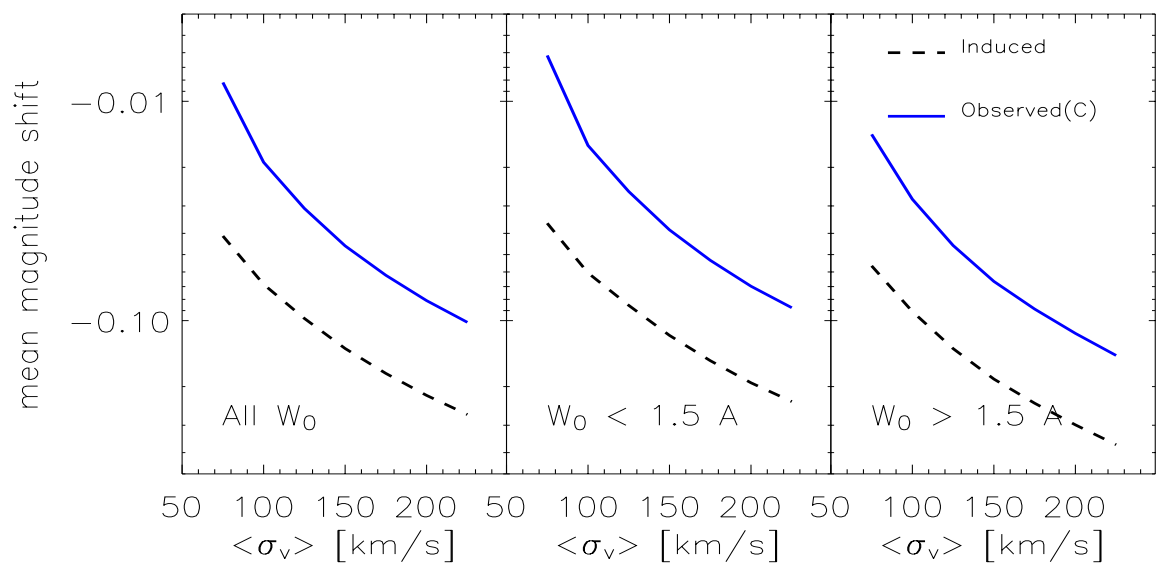

Figure 2. Mean magnitude shifts of quasars due to magnification by intervening MgII absorbers at $z=0.6$, as a function of the mean velocity dispersion of the absorbing galaxies. The dashed curve shows the real mean magnitude shift induced and the colored curve shows the mean shift that is observed, as a result of the magnification bias. We have computed the signal expected for the completeness function introduced in the text. The left panel shows the effect for absorbers with $0.3<W_{0}<3.3 \AA$. The middle and right panels show how the signal is expected to vary as a function of rest equivalent width $W_{0}$.

suggest to use metal absorbers because they allow for the compilation of larger samples and they can be detected from the ground over favorable redshift ranges for lensing.

Due to magnification effects, a given population of absorbers will give rise to a distribution of induced quasar magnitude changes, which is conveniently described by its centered moments $\left\langle\delta m^{i}\right\rangle$. The corresponding magnitude changes that can be observed, $\Delta\left\langle m_{\text {obs }}^{i}\right\rangle=\left\langle m_{\text {lens }}^{i}\right\rangle-\left\langle m_{\text {ref }}^{i}\right\rangle$, depend on the shape of the quasar luminosity function and the completeness of the survey. We have focused on the first-order moment and we have shown how the observed magnitude changes $\Delta\left\langle m_{\text {obs }}\right\rangle$ can be used to recover the induced ones $\langle\delta m\rangle$ and then constrain the characteristic mass of the absorbing galaxies.

Our method can be applied to various absorption lines (MgII, FeII, SiII, CIV, etc.). In order to explore its feasibility, we have computed the expected lensing effects for a population of MgII absorbers. Using the observed distribution of rest equivalent widths as a function of impact parameter measured by Steidel et al.(1994), and assuming that the mass profile of absorber systems is described by that of an isothermal sphere with a mean velocity dispersion $100<\left\langle\sigma_{v}\right\rangle<200 \mathrm{~km} \mathrm{~s}^{-1}$, we have shown that: quasars behind MgII absorbers with $0.3<W_{0}<1.5 \AA$ are in average brighter by $\langle\delta m\rangle=-0.03$ to -0.1 magnitude. For systems with $1.5<W_{0}<3.2 \AA$ these values reach -0.1 to -0.4 magnitude.

Using the quasar luminosity distribution based on the 2dF Quasar survey (Croom et al. 2004) and realistic completeness functions we have shown that the corresponding magnitude changes that can be observed are $\Delta\left\langle m_{\text {obs }}\right\rangle=-0.006$ to -0.08 for systems with $0.3<W_{0}<1.5 \AA$, and -0.014 to -0.14 in the range $1.5<W_{0}<3.2 \AA$.

Whereas our model provides us with estimates of the unavoidable magnitude changes $\left\langle\delta m^{i}\right\rangle$ due to magnification, it does not allow us to determine, in the general case, the 
detectability of $\Delta\left\langle m_{\text {obs }}^{i}\right\rangle$ since effects of extinction by dust need to be included. Observing at sufficiently large wavelengths can however isolate the lensing effects.

In addition to probing the mass distribution of absorber systems, our analysis suggests that quasars behind metal absorbers are not necessarily dimmed, as is usually mentioned in the literature, but they can actually be brighter if extinction effects are smaller than the gravitational magnification effects presented above. The claim that magnitude limited surveys might miss a number of quasars with strong absorbers must therefore be revisited. Applying our method to large surveys will allow us to quantify this effect.

\section{References}

Bartelmann, M. \& Loeb, A., 1996, ApJ, 457, 529

Boyle, B. J., Shanks, T., Croom, S. M., Smith, R. J., Miller, L., Loaring, N., \& Heymans, C. 2000, MNRAS, 317,

Croom, S. M., Smith, R. J., Boyle, B. J., Shanks, T., Miller, L., Outram, P. J., \& Loaring, N. S. 2004, MNRAS, 349, 1397

Ellison, S. L., Ibata, R., Pettini, M., Lewis, G. F., Aracil, B., Petitjean, P., \& Srianand, R. 2004, A\&A, 414, 79

Hamana, T., Martel, H., \& Futamase, T. 2000, ApJ, 529, 56

Kobayashi, N., Terada, H., Goto, M., \& Tokunaga, A. 2002, ApJ, 569, 676

Le Brun, V., Smette, A., Surdej, J. \& Claeskens, J.-F., 2000, A\&A 363, 837L

Maller, A. H., Kolatt, T. S., Bartelmann, M. \& Blumenthal, G. R., 2002, ApJ, 569, 72

Ménard, B. and Péroux, C., 2003, A\&A 410, 43

Ménard, B., Nestor, D. \& Turnshek, D., in preparation

Myers, A. D., Outram, P. J., Shanks, T., Boyle, B. J., Croom, S. M., Loaring, N. S., Miller, L., \& Smith, R. J. 2003, MNRAS, 342, 467

Pei, Y. C., 1995, ApJ, 440, 485

Perna, R., Loeb, A \& Bartelmann, M., 1997, ApJ 448, 550

Smette, A., Robertson, J. G., Shaver, P. A., Reimers, D., Wisotzki, L., \& Koehler, T. 1995, A\&As, 113, 199

Smette, A., Claeskens, J. F. \& Surdej, J., 1997, New Astronomy 2, 53

Steidel, C. C., Dickinson, M. \& Persson, S. E., 1994, ApJ, 437, L75

Steidel, C. C. 1995, astro-ph/9509098 